\title{
Optical imaging of the molecular mobility of single polystyrene nanospheres
}

Shasha Liư ${ }^{1}$, Mengqi Lv${ }^{1}$, Haoran $\mathrm{Li}^{1}$, Sa Wang ${ }^{1}$, Chengdong Feng ${ }^{2}$, Xiaoliang Wang², Wenbing $\mathrm{Hu}^{2}$, Wei Wang ${ }^{1 *}$

${ }^{1}$ State Key Laboratory of Analytical Chemistry for Life Science, Chemistry and Biomedicine Innovation Center (ChemBIC), School of Chemistry and Chemical Engineering, Nanjing University, Nanjing 210023, China

2 State Key Lab of Coordination Chemistry, Department of Polymer Science and Engineering, School of Chemistry and Chemical Engineering, Nanjing University, Nanjing 210023, China

*To whom correspondence should be addressed.

Email: wei.wang@nju.edu.cn 


\section{Table of Contents}

S1. Detailed geometry and thermal controllability of gold film

S2. Build the calibration between the electric power and the temperature of gold film

S3. Temperature stability evaluated by time-dependent electrical power fluctuation

S4. Temperature homogeneity in gold film

S5. Calibration of optical intensity with the size of PSNS

S6. Comparing the optical response of single PSNS with DSC results of bulk sample

S7. SEM and AFM characterizations of PSNS before and after glass transition

S8. Optical scattering intensity of single PSNS simulated by discrete dipole approximation method

59. The different optical response between glass transition and rubber-fluid transition process

S10. The method for extracting the $T_{\mathrm{g}}$ of PSNS

S11. The method for extracting the $T_{\mathrm{f}}$ of PSNS

S12. Comparing the $T_{\mathrm{f}}$ response of 3 PSNS shown in Figure 4

S13. Detailed geometry and characterization of used $\mathrm{SiO}_{2}$ substrate

S14. The $T_{\mathrm{g}}$ and $T_{\mathrm{f}}$ response of PSNS under different temperature scan rates

S15. Detailed procedures for extracting the $\Delta I_{1}$ and $\Delta I_{2}$ of each PS nanoparticles

S16. Optical response of all 56 PS nanoparticles in the imaging field of view

Movie S1. Comprehensive movie for exhibiting the optical response of PSNS during heating process

Movie S2. Dynamic rubbery flow process of PSNS on the gold film 


\section{S1. Detailed geometry and thermal controllability of gold film}

In order to heat the polystyrene nanospheres (PSNS) effectively, the gold film with thickness of 50-nm was sputtered on the coverslip. More importantly, the gold film was designed as such special pattern to reduce the influence of large-area heat source on the optical imaging system. The gold band (red rectangle) in the middle was the effective heating area (i.e., resistor) owing to the small cross-sectional area according to the theory of Joule law.

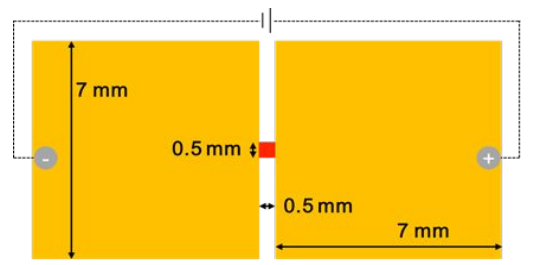

Figure S1. Geometry of the gold film used in the experiment.

The voltage applied on the gold film was realized by an electrochemical workstation, and the value of voltage as well as the scan rate was controlled by the wave function generator just as shown in Figure 1a. The non-linear time dependent voltage curve (Figure 1b, black line) was pretyped into the wave function generator, and the temperature scan rate can be easily adjusted by changing the output frequency in wave function generator.

\section{S2. Build the calibration between the electric power and the temperature of gold film}

In order to build the calibration between the applied electric power and the temperature of gold band, four pure materials, lauric acid, palmitic acid, arachidic acid and KSCN, were heated under the same condition with PSNS. A pipette with diameter of $10 \mu \mathrm{m}$ was employed to deposit the four materials on the band of gold film. As expected, the four materials melted in order according to the melting point under electrical power ramping. By correlating the melting point with required electrical powers for melting of the four materials (red dots in Figure 1c), the calibration between the temperature of band and applied electric power was obtained and shown in Figure 1c (black dash line).

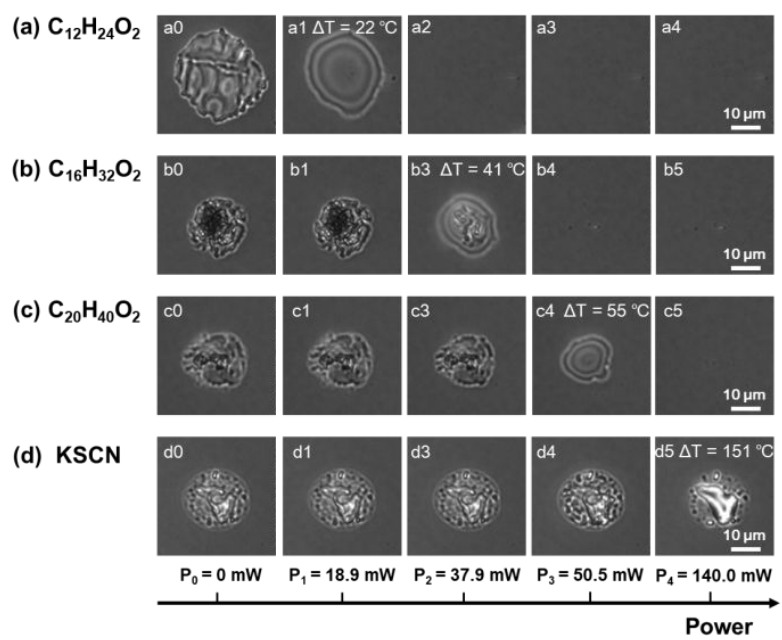

Figure S2. Snapshots of four substances during the heating process. 


\section{S3. Temperature stability evaluated by time-dependent electrical power fluctuation}

In order to access the temperature stability, the electrical power as a function of time was studied under constant voltage of $0.8 \mathrm{~V}$. The mean response electrical power was $94.5 \mathrm{~mW}$ with standard deviation of $0.1 \mathrm{~mW}$ in $30 \mathrm{~min}$, and the deviation of temperature fluctuation was deduced to be $0.1^{\circ} \mathrm{C}$ according to the calibration between electrical power and temperature of gold band.

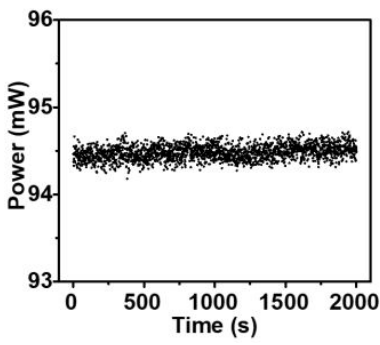

Figure S3. The electrical power curve as a function of time under the constant voltage of $0.8 \mathrm{~V}$.

\section{S4. Temperature homogeneity of gold band}

In order to access the homogeneity of temperature distribution in the band of gold film, the thermal response of 94 PSNS with uniform size (purchased from Aladdin) in one experiment were accessed. The scatter diagram in Figure S4b exhibited the final $T_{\mathrm{g}}$ of each PSNS in the field of view as a function of the radius. Statistics (blue bar) and fitting (black curve) result shown that the $T_{\mathrm{g}}$ at $106.1^{\circ} \mathrm{C}$ with a standard deviation of $2.5^{\circ} \mathrm{C}$. Note that the calculated heterogeneous temperature distribution of $T_{\mathrm{g}}$ also included the difference between PSNS, hence the temperature variation of gold band itself should be smaller than $2.5^{\circ} \mathrm{C}$.
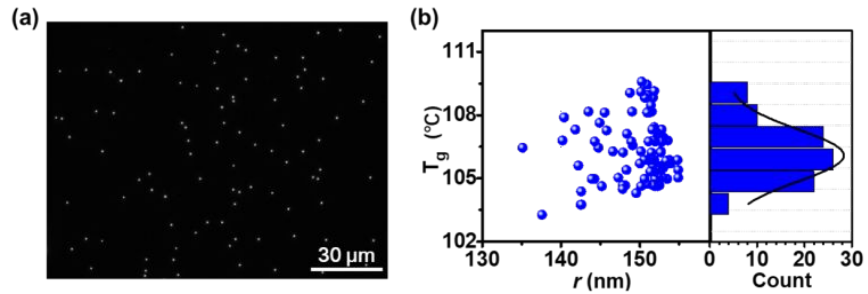

Figure S4. (a) DFM image of 94 individual PS on the band of gold film. (b) Determined $T_{\mathrm{g}}$ of all 94 PSNS in the (a) as a function of its corresponding radius as well as the statistics results.

\section{S5. Calibration of optical intensity with the size of PSNS}

In order to deduce the size of PSNS from the DFM images, the calibration between original optical intensity and size of PSNS was established. After the DFM image acquired from the optical imaging system, the PSNS in the very same area was then characterized by SEM. Corresponding radius and the optical intensity of the every and each PSNS in the field were acquired and plotted in Figure S5c, where each black dot represent a single PSNS and the red dash line shown the final fitting result. Results revealed that the optical scattering intensity decreased as a power factor of six with the reducing size of PSNS. 
(a)

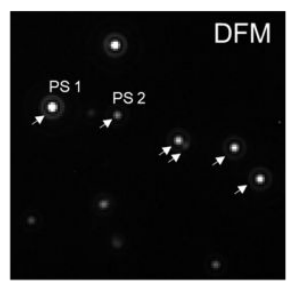

(b)

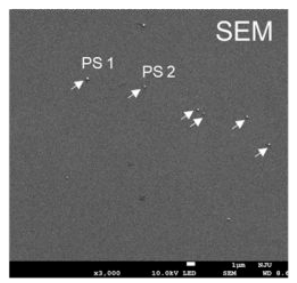

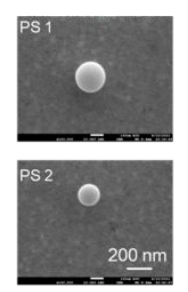

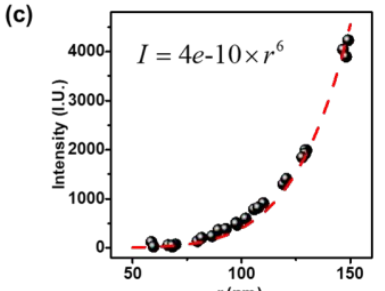

Figure S5. The DFM image (a, for extracting the optical intensity of PSNS) and correlated SEM image (b, for extracting the size of PSNS) of the same field used for calibration. Enlarged SEM images exhibited two typical PS nanospheres in the area for demonstrating that each bright spot in DFM indeed represented one single PSNS. (c) Optical intensity versus corresponding radius of 27 PSNS. And the red line shown the final fitting result of the calibration between optical intensity and size of the PSNS.

\section{S6. Comparing the optical response of single PSNS with DSC results of bulk sample}

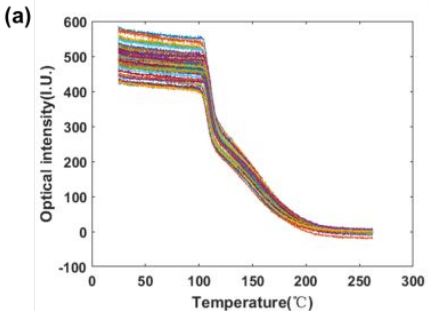

(b)

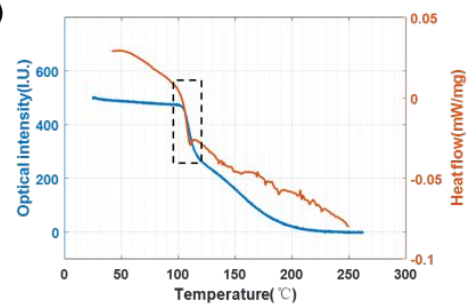

Figure S6. (a) The optical response of all 56 PS individuals in the field of view. (b) The mean optical response of all PSNS was shown in blue lines, and the DSC characterization result of $2.1 \mathrm{mg}$ bulk PS powder with temperature scan rate of $10^{\circ} \mathrm{C} / \mathrm{min}$ was exhibited as orange line. The obvious change of heat flow in DSC result were in good agreement with the first descend step in the optical response.

\section{S7. The SEM and AFM characterizations of PSNS before and after glass transition}
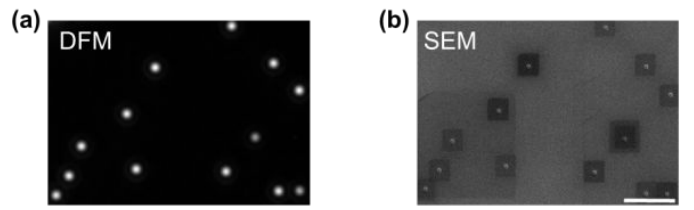

(c)
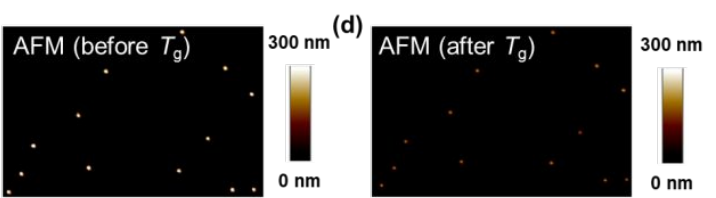

Figure S7. (a) DFM image of PSNS acquired before heating. (b) SEM image of the very same PSNS after the glass transition for diameter characterization. AFM images of the same PSNS before (c) and after (d) glass transition for height characterization. The PSNS used here were 300-nm PSNS purchased from Aladdin.

\section{S8. Optical scattering intensity of single PSNS simulated by discrete dipole approximation method}

The optical scattering intensity of a single PSNS was regulated by some of its properties including size, refractive index and morphology. During the glass-rubber transition process, the thermal 
induced expansion, reduced refractive index and morphological deviation all would influence the optical scattering intensity of PSNS, as discussed in detail below.

1) Thermal expansion. According to 'Polymer Handbook' (Fourth Edition, edited by Brandrup et al), the coefficient of thermal expansion (volume) was $\sim 2 \times 10^{-4} \mathrm{~K}^{-1}$ (glassy state). ${ }^{1}$ The temperature ramping from 25 to $105{ }^{\circ} \mathrm{C}$ accordingly increase the volume by $1.6 \%$, and thus the scattering intensity by $3.2 \%\left(I \propto \mathrm{V}^{2}\right.$ or $\left.\mathrm{R}^{6}\right)$.

2) Refractive index. Ward and coworkers recently used optical scattering to systematically determine the refractive index of polystyrene beads as a function of temperature. ${ }^{2}$ The temperature ramping from 25 to $105{ }^{\circ} \mathrm{C}$ was found to reduce the refractive index from 1.597 to 1.581 , corresponding to a decrease in the scattering intensity by $\sim 4.3 \%$ according to the Rayleigh scattering theory. ${ }^{3}$

3) Morphology. The scattering is dependent on not only the size and refractive index, but also the morphology of the nanoparticle. AFM and SEM characterizations of 13 PSNS (shown in Figure 2 and Figure 57) provided clear evidences to show that, the morphology of PSNS changed from the sphere with $r_{s}=150 \mathrm{~nm}$ to a hemisphere of approximately equal volume with $r_{h s}=189 \mathrm{~nm}$.

In order to compare the optical scattering intensity of PSNS in the two above-mentioned situations, the discrete dipole approximation method (which was realized by DDSCAT V7.3) was used to evaluate the scattering efficiency factor (Q) of the sphere with the refractive of $n_{s}=1.597\left(R_{\text {eff }}=\right.$ $150 \mathrm{~nm})$ and a hemisphere of equal volume with the refractive of $n_{h s}=1.581\left(R_{\text {eff }}=150 \mathrm{~nm}\right)$ under the same illumination condition (wavelength $=520 \mathrm{~nm}$ ), respectively. As shown in Figure 58 below, the results indicated that, even though the volumes were similar, the scattering efficiency factor of the hemisphere $\left(Q_{h s}=1.42\right)$ was about $30 \%$ smaller than that of the sphere $\left(Q_{s}=2.09\right)$. It was in good agreement with the observed decreased optical intensity in the experiment.

(a)

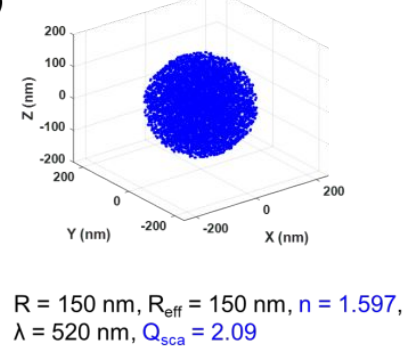

(b)

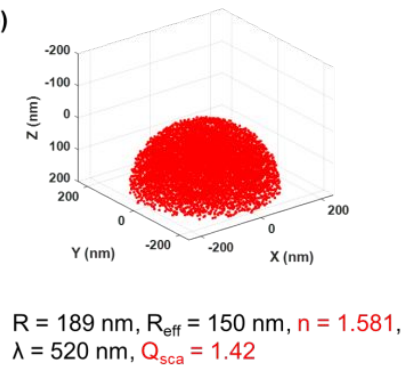

Figure S8. DDA simulation results of single PSNS before (a) and after (b) glass transition.

\section{The different optical response between glass transition and rubber-fluid transition process}

If a constant temperature of $112.6{ }^{\circ} \mathrm{C}$ was applied to the surface and it was kept for about 4 minutes, the optical intensity of single PSNS was found to be stable, suggesting the morphological stability of PSNS at this stage. This feature was consistent with the softening. Note that the softening was different from the flow behavior at higher temperature $T_{\mathrm{f}}$, because the latter was accompanied with the persistent spreading of the object and thus the gradual decrease in the optical intensity until disappearance. 


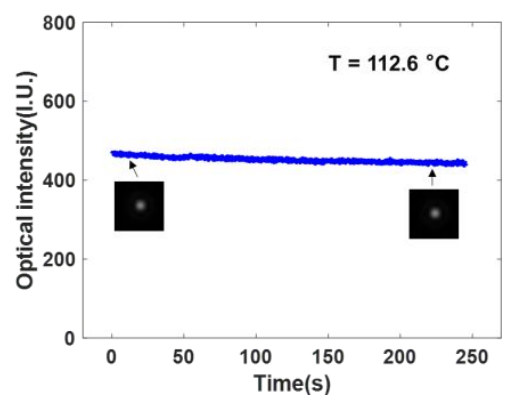

Figure S9. The optical intensity of single PSNS was found to be stable under the constant temperature of $112.6^{\circ} \mathrm{C}$ (slightly higher than $T_{\mathrm{g}}$ ).

\section{S10. The method for extracting the $T_{\mathrm{g}}$ of PSNS}

Firstly, the $\mathrm{dl} / \mathrm{dt}$ response curve of each PSNS was obtained by differential the smoothed temperature-optical intensity curve. Then the $T_{\mathrm{g}}$ was confirmed by finding the temperature where the minimum $\mathrm{dl} / \mathrm{dt}$ value located in.

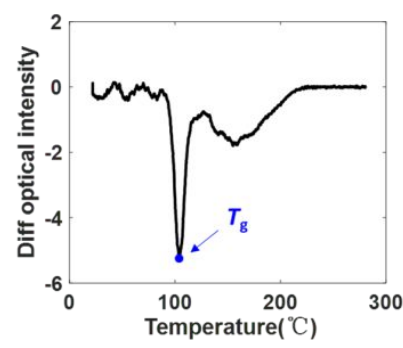

Figure S10. The $\mathrm{dl} / \mathrm{dt}$ curve as function of temperature of one typical PSNS in the field of view, and the blue point indicated the obtained $T_{\mathrm{g}}$ for this PSNS.

\section{S11. The method for extracting the $T_{\mathrm{f}}$ of PSNS}

Upon heating, the PSNS finally turn into a thin film, which beyond the detection limitation of DFM. Therefore, we defined the temperature when the optical intensity dropped to the 10 times of the noise level higher than the baseline (indicated as blue dash line) as the $T_{f}$ of the PS nanospheres. The precise time (temperature) for $T_{f}$ was finally determined by fitting the time-optical intensity curve with polynomial function and find the intersection point between thresholds and the time - optical intensity curve.

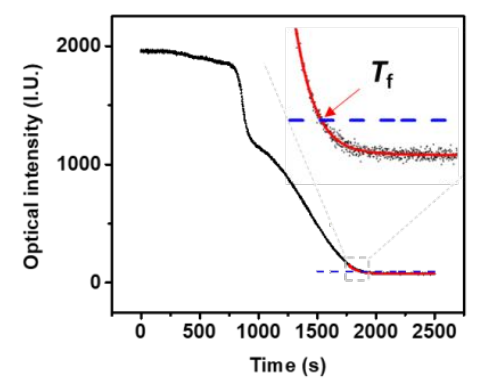

Figure S11. The optical intensity curve as function of time of one PSNS in the field of view. The red line shown the fitting result, and the blue dash line indicated the calculated threshold for determining the $T_{\mathrm{f}}$ of PSNS. 


\section{S12. Comparing the $T_{\mathrm{f}}$ response of 3 PSNS shown in Figure 4}
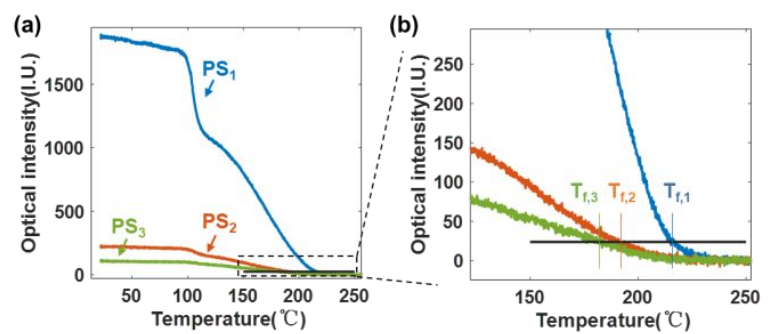

Figure S12. The obtained $T_{\mathrm{f}}$ of PSNS with different size shown in Fig. 4a-c.

\section{S13. Detailed geometry and characterization of used $\mathrm{SiO}_{2}$ substrate}

In order to make a $\mathrm{SiO}_{2}$ surface, at the same time, it could heat the PSNS placed on the surface effectively, herein a $\mathrm{SiO}_{2}$ layer was sputtered on top of the previously-used $\mathrm{Au}$ substrate. The thickness of $\mathrm{SiO}_{2}$ was about $25 \mathrm{~nm}$, which was characterized by Stylus profiler.

(a)

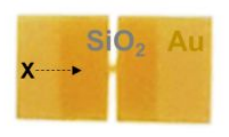

(b)

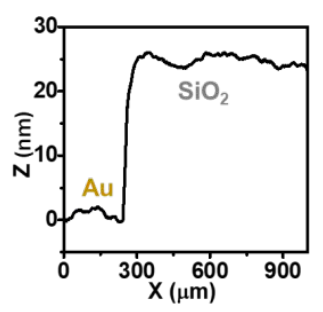

Figure S13. (a) The image of $\mathrm{SiO}_{2}$ substrate used in experiment, where the band (effective heating area) was totally covered with $\mathrm{SiO}_{2}$ layer. (b) The height profile of substrate along the black dash line shown in (a).

\section{S14. The $T_{\mathrm{g}}$ and $T_{\mathrm{f}}$ response of PSNS under different temperature ramping rate}

The $T_{\mathrm{g}}$ and $T_{\mathrm{f}}$ response of PSNS under different temperature ramping rates $\left(35.9^{\circ} \mathrm{C} / \mathrm{min}\right.$, $18.2^{\circ} \mathrm{C} / \mathrm{min}$ and $7.2^{\circ} \mathrm{C} / \mathrm{min}$ ) was studied. It was found that, both the $T_{\mathrm{g}}$ and $T_{\mathrm{f}}$ increased as the temperature scan rate increased (Figure S14a-b), $T_{f}$ showed a more evident dependence on the temperature scan rate, indicating that the rubber-fluid transition was a slower dynamic process than the glass transition. For single PSNS with similar sizes, much higher apparent $T_{\mathrm{f}}$ was observed at larger temperature ramping rate (Figure S14c). In addition, weaker dependence of $T_{\mathrm{f}}$ with size was revealed at slower temperature ramping (Figure $\mathrm{S} 14 \mathrm{~b})$. These results clearly demonstrated that the higher apparent $T_{\mathrm{f}}$ was a result of slower rubber-fluid transition kinetics in larger PSNS.

Based on the experimental results, we clarify that the obtained $T_{\mathrm{f}}$ values of single PSNS was apparent $T_{\mathrm{f}}$, which was not only determined by the intrinsic property (such as molecular weight), but also the kinetic factors (such as the temperature scan rate). 

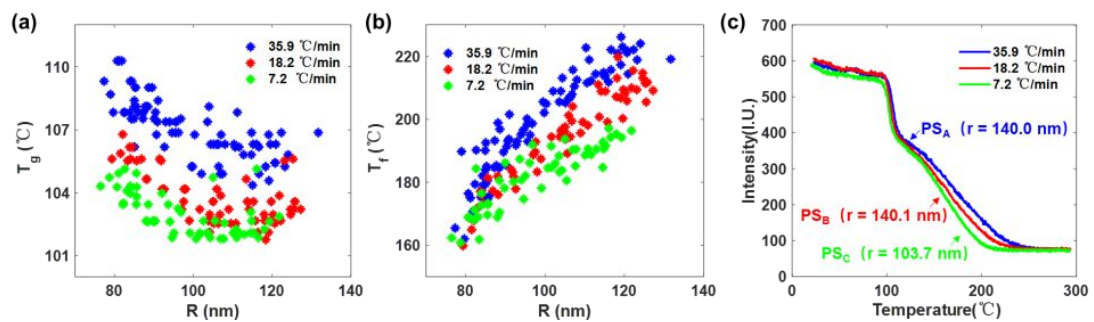

Figure S14. The $T_{\mathrm{g}}(\mathrm{a})$ and $T_{\mathrm{f}}(\mathrm{b})$ response of PSNS under different temperature ramping rates. (c) The optical response of PSNS with similar size under different temperature scan rates.

\section{S15. Detailed procedures for extracting the $\Delta \mathrm{I}_{1}$ and $\Delta \mathrm{I}_{2}$ of each PS nanoparticles}

In order to extracting the $\Delta \mathrm{I}_{1}$ and $\Delta \mathrm{I}_{2}$ from the time-optical intensity curve of single PS nanospheres, three points should be determined, i.e., the beginning point of $\Delta \mathrm{l}_{1}\left(\mathrm{P}_{0}\right)$, the ending point of $\Delta I_{1}\left(P_{1}\right)$, and the ending point of $\Delta I_{2}\left(P_{2}\right)$, which were indicated as red star in Figure 4a. Combined the response of optical intensity and the thermal transition process of PS, the turning point on the intensity curve should be the beginning or the ending of one process. Therefore, the turning point, i.e., the point where the slope of curve changed was the goal for searching. The beginning point $\left(P_{0}\right)$ of $\Delta \mathrm{l}_{1}$ was easily determined as the point where the optical intensity started to decrease. We than calculated the slope between all points and $P_{0}$, and the result was shown in Figure S15b. A clear turning at about $770 \mathrm{~s}$ was observed. In order to further extract the accurate turning point $P_{1}$, the slope between each point and $P_{0}$ were then fitted by a polynomial function (blue line in Figure $S 15 b$ ), finally the turning point was determined as the intersectional point between threshold (10 times standard deviation of data from $700 \mathrm{~s}$ to $750 \mathrm{~s}$ below the mean value of data from $700 \mathrm{~s}$ to $750 \mathrm{~s}$, indicated as red dash line) and time dependent slope curve. The obtained turning point $\left(P_{1}\right)$ was indicated by red arrow in Figure $S 15 b$. The turning point $P_{2}$ was determined by the similar way.

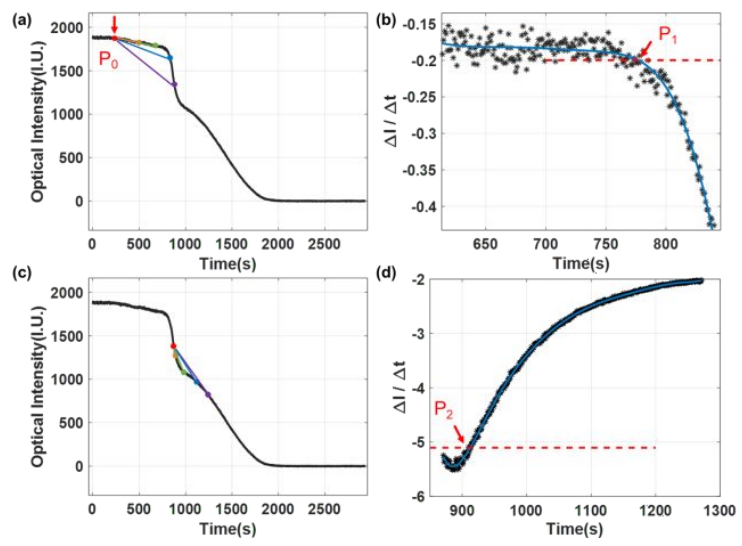

Figure S15. (a) Time-dependent optical intensity response of one PSNS. The red point located was determined as the beginning point of $\Delta \mathrm{l}_{1}$, i.e., $\mathrm{P}_{0}$. The yellow, green, blue and purple dots were selected to show how the slope was calculated and changed as time. (b) Calculated slope in (a) of points during 500-900 $\mathrm{s}$. The blue line shown the fitting result and the red dash line indicated the threshold for determining turning point, $P_{1}$. (c) Method for calculating the slope for determining $P_{2}$. (d) 
Calculated slope in (c) of points during 850-1300 s. The blue line shown the fitting result, and the red dash line indicated the threshold for determining turning point, $\mathrm{P}_{2}$.

\section{S16. Optical response of all 56 PS nanoparticles in the imaging field of view}

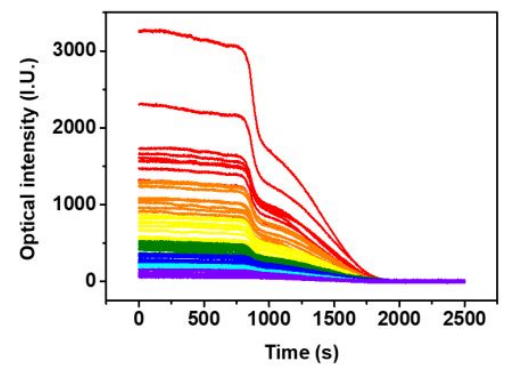

Figure S16. The optical response of all 56 PS nanospheres in once experiment.

Movie S1. Comprehensive movie for exhibiting the optical response of PSNS during the heating process.

Movie S2. Dynamic flow process of PSNS on the gold film.

\section{Reference}

1. J. Brandrup, E. H. Immergut, E. A. Grulke. Polymer Handbook, 4th Edition.

2. McGrory MR, King MD, Ward AD. Using Mie Scattering to Determine the Wavelength-Dependent Refractive Index of Polystyrene Beads with Changing Temperature. J. Phys. Chem. A, 2020, 124, 9617-9625.

3. Zhu, S., Ma, L., Wang, S., Yan, X., Light-Scattering Detection below the Level of Single Fluorescent Molecules for High-Resolution Characterization of Functional Nanoparticles. ACS Nano, 2014, 8, 10998-11006.

4. Draine, B. T., Flatau, P. J., Discrete-dipole approximation for scattering calculations. J. Opt.Soc. Am., 11, 1491-1499.J. Opt. Soc. Am. A, 1994, 11, 1491-1499. 